\title{
Diversity and distribution of figs (Ficus: Moraceae) in Gianyar District, Bali, Indonesia
}

\author{
I MADE SAKA WIJAYA", MADE RIA DEFIANI \\ Department of Biology, Faculty of Mathematics and Natural Sciences, Universitas Udayana. Jl. Raya Kampus Unud, Jimbaran, Badung 80361, Bali, \\ Indonesia. Tel.: +62-361-701954, •email: sakawijaya @unud.ac.id
}

Manuscript received: 3 November 2020. Revision accepted: 21 December 2020.

\begin{abstract}
Wijaya IDS, Defiani MR. 2021. Diversity and distribution of figs (Ficus: Moraceae) in Gianyar District, Bali, Indonesia. Biodiversitas 22: 233-246. Fig (Ficus) is an important species in tropical regions, but there is a lack of updated data on fig diversity in Bali. This study of fig diversity and distribution in Gianyar District, Bali, Indonesia was conducted to record the alpha-diversity of figs. The intensive exploration method was applied at seven locations representing the seven sub-districts of Gianyar. Habitats and coordinates were recorded, and the appropriate samples were preserved as dried or spirit specimens. The results showed that there were 23 identified species of figs from six subgenera: Ficus (1), Pharmacosycea (2), Sycidium (5), Sycomorus (5), Synoecia (2), and Urostigma (9). Based on location, three species had restricted distribution: $F$. fulva, F. tinctoria ssp. globosa, $F$. virens, and $F$. subcordata, whereas six species were adapted in every location: F. montana, F. hispida, F. septica, F. pumila, F. benjamina, and F. superba. The figs were primarily distributed near bodies of water, especially riverbanks.
\end{abstract}

Keywords: Ara, fig, hemi-epiphyte, keystone species, syconium

\section{INTRODUCTION}

Indonesia is a tropical country which biogeographically comprised of the Sundaland and Wallacea regions. Those regions are biodiversity hotspots with conservation priority that had more than 1,500 endemic plants and animals, but have lost $75 \%$ of their primary vegetation (Myers et al. 2000). Forest is a terrestrial ecosystem that preserves the biodiversity in Indonesia, however, it was the area with highest loss by deforestation. As an ecosystem, the forest had complex interactions among its organisms, but this complexity could be simplified by studying the keystone species. Keystone species maintain the ecosystem's stability by altering the species composition. It could be a top predator, a dispersal agent, or a tree whose importance is rarely noticed, such as the fig (Ficus).

Fig (Ficus: Moraceae) is abundant in tropical regions and around 367 out of 735 species were growing in Malesia (Berg and Corner 2005). The high diversity among figs includes their various growth forms. Trees or shrubs were the most dominant growth forms (also called the foresttype), whereas the other growth forms were lianas or creeping figs (about 100 species) and hemi-epiphytes (about 300 species) (Berg and Corner 2005). Hemiepiphytes are epiphytic plants that require host plants to attach to. When they grow, the roots would reach the soil through the surface stem of the host tree and then gradually grow bigger until the host is strangled (van Steenis 1972; Harrison et al. 2003). A hemi-epiphyte is also known as an intricate growth form because its successional rate depends on many factors, such as the species' ability, the size of the host tree, and the position in the canopy in terms of access to light (Harrison et al. 2003).
Figs can be distinguished from other plants by their syconium. There has been some confusion because the term "fig" could refer to either the fig as an entire plant or its syconium. The fig's syconium is a hollow, fleshy receptacle with only one hole at the tip called ostiole or orifice, which is enclosed by multiple inner brachtea. Inside the syconium, there are unisexual apetal flowers. Each female flower bears one ovule. During pollination periods, the syconium will release a species-specific compound to attract certain pollinators (Harrison and Rasplus 2006; Wang et al. 2013), and then the inner-brachtea will stretch to form a small path for the pollinator (Harrison and Rasplus 2006).

Ficus is an important genus in tropical forests because it maintains the ecosystem's stability by providing food for many types of frugivores ( Shanahan et al. 2001; Harrison 2005; Sreekar et al. 2010; Kuaraksa et al. 2012). Figs are capable of producing a large number of fruits, and most of the species can bear fruit throughout the year, making them a potential resource for frugivores in periods of scarcity (Shanahan et al. 2001; Wang et al. 2005; Walther et al. 2017). Most of the bats in the subfamily Stenodermatinae are fig specialists because they rely on figs as a keystone resource (Bravo et al. 2012). The more figs are eaten, the wider the distribution of their seeds, whether by endozoochory, dys-zoochory, or exo-zoochory that related to the frugivores dispersal (Harrison 2005). Ultimately, this seed dispersal has played a key role in defining the forest's composition and accelerating colonization in a phase of succession by transferring seeds from the nearby island (Wandrag et al. 2017). There is evidence that the dispersal and ecological factors were the keys to establishing the current fig distribution (Xu et al. 2015). Ficus species were 
recorded in a sere of succession on Krakatau Island (after the massive explosion in 1883) as a keystone species in forest diversification through fig-frugivore interaction (Thornton et al. 1996). Thus, indirectly, the appearance of figs could be used as an assessment material to inspect the ecosystem's health or restoration progress (Sreekar et al. 2010; Cottee-Jones et al. 2016). In urban environments, native figs have proven to be a vital resource to maintain biodiversity (Lok et al. 2013; Lim et al. 2017; Walther et al. 2017).

Fig has specific interactions with insect pollinators (generally Aganoidae), which can explain the coevolution mechanism through species-specific pollinators (Harrison and Rasplus, 2006; Cruaud et al. 2012; Suleman et al. 2014). Gall flowers in figs are useful for Aganoidae insect to oviposit their eggs as a reciprocal reward for pollination (Suleman et al. 2014). If the pollinator community dropped excessively, it would affect the interaction patterns of figs/fig-pollinating wasps interaction to host/parasite interaction by non-pollinator insects (Wang et al. 2005; Zhang et al. 2020), or it could cause the occurrence of a host shifting phenomenon by non-pollinator insects, leading to hybridization (Tsai et al. 2015; Zhang et al. 2020). Cruaud et al. (2012) published comprehensive work on fig/fig-pollinating wasp interaction and found that if the mutualistic interaction was a long-term codiversification that spread from Eurasia. The codiversification might be included in major evolutionary events in figs. Zhang et al. (2019) hypothesized that the fig/fig-wasp interaction might be one of the factors that led to several fluctuations in the evolution of monoecious and dioecious figs, which is connected with the fig's recent biogeographical range. Biogeographical range correlated with gene flow could be mismatching some characters or strengthening the coadaptation pattern (Medeiros et al. 2018).

Figs have proven to be an essential part of the tropical ecosystem, but there has been a lack of updated data on fig diversity in Bali. The latest data only show the fig diversity of Lesser Sunda with 34 species (Berg and Corner 2005). Harrison (2005) stated that diversity (alpha-diversity) of the fig should be re-assessed because it might be underestimated due to the sampling protocol. Fig diversity was derived from a tree plot, however, there were not many figs with the tree growth form in the lowland forest (Harrison 2005).

The Balinese culture mostly integrated with Hinduism used many species of plants in religious offerings which is indirectly lead to local-based conservation (Sujarwo et al. 2020). The use of figs in Balinese culture is not the only factor that causes the existence of figs in Bali. A more influential factor is the natural environment conditions that suit the figs' habitat, one of which is in Gianyar District. This district has various altitudes from 0-950 m asl. (BPSStatistics of Gianyar 2019) with diverse types of landscapes such as coasts, estuaries, lowland areas, and highland areas. This district also has many permanent rivers that flow throughout the year (Hadisusanto et al. 2018). Watersheds, especially riparian vegetation or riverbanks are the habitat preference for figs. Based on those factors, this study aimed to assess the diversity and distribution of figs in Gianyar.

\section{MATERIALS AND METHODS}

\section{Study area}

Research was conducted from March to September 2020 in Gianyar District, Bali, Indonesia. This district is located at S: $08^{\circ} 11^{\prime} 48^{\prime \prime}-08^{\circ} 38^{\prime} 58^{\prime \prime}$ and E: $115^{\circ} 13^{\prime} 29^{\prime \prime}-$ $115^{\circ} 22^{\prime} 23^{\prime \prime}$, covers 36,800 ha area, and has an altitude varying from 0-950 m asl. (BPS-Statistics of Gianyar 2019). Gianyar District consists of 7 sub-districts which were used as the 7 main exploration areas: I (Blahbatuh), II (Gianyar), III (Sukawati), IV (Ubud), V (Payangan), VI (Tampaksiring), and VII (Tegallalang), as shown in Figure 1.

\section{Data collection, preparation of specimens, and identification}

Morphological characterization was carried out by describing the characteristics of figs such as roots, stems, leaves, flowers, and fruits. Each characteristic was photographed/documented, particularly the fruit (syconium), which is known as the main feature of the fig. The syconium was split longitudinally in order to obtain the structure of the orifice, flower arrangement, color, and characteristics of the fleshy receptacle. Fruit characteristics could indicate the preferences of frugivores based on size, color, and fruit location (Shanahan et al. 2001). Supporting descriptions were also added, such as their habitat, habitus or growth form, and interactions with other organisms were included as supplementary taxonomical data.

The fruit (syconium) is the most important organ of the fig species, and it should be prioritized in sample collection (Rugayah et al. 2004). The fruit was preserved as a spirit specimen by putting it in a jar and adding $70 \%$ alcohol, closing the jar tightly, and labelling it. Other samples such as leafy twigs with or without fruit, were preserved as dry specimens according to Womersley (1981). Samples were washed in running water, placed in papers, and sprayed with $70 \%$ alcohol for sterilization. Samples were stacked in group of 5-10, then pressed with plywood to dry in an oven for about 7 days. After being dried, samples were arranged on 420 x $297 \mathrm{~cm}$ paper, labeled, and covered with transparent plastic. Labels consisted of much information, such as the collection number, collector, collection date, family, scientific name, local name, determinator, date of identification, location, habitat, and annotations composed of description of characteristics that could not be collected or probably change during preservation (Womersley 1981; Rugayah et al. 2004). The collected spirit and dried specimens (voucher specimens) were stored at the Biology Study Program, Faculty of Mathematics and Natural Sciences, Udayana University, Bali, and not yet deposited in any herbarium. 


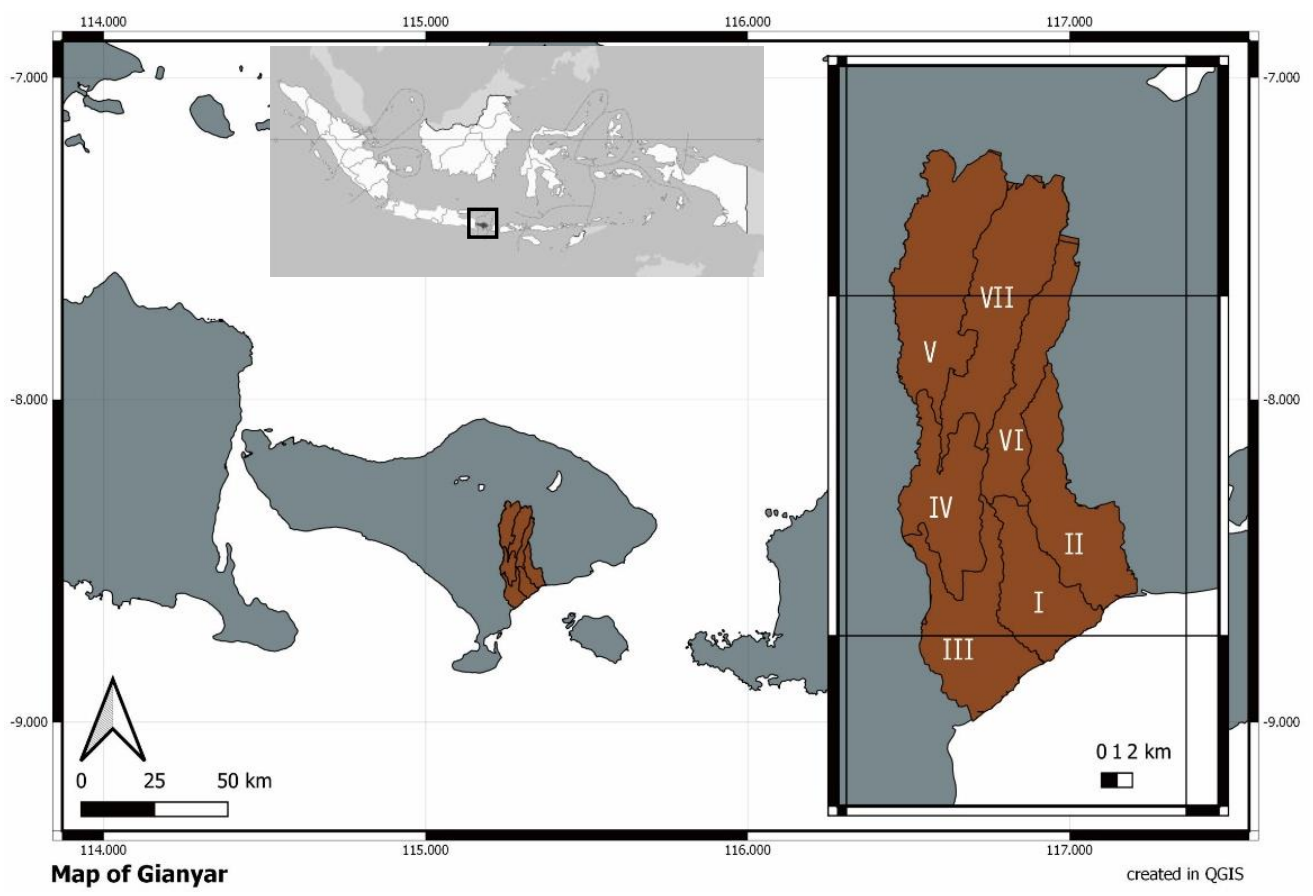

Figure 1. Exploration area for figs (Ficus) in Gianyar, Bali, Indonesia

Photos, spirit specimens, and dried specimens that collected were important materials for identification. The books Flora of Java vol. 2 (Backer and van den Brink, 1965) and Flora Malesiana series 1 Spermatophyta, Moraceae: Ficeae (Berg and Corner 2005) were used as the two main references for identification, while for nomenclature status was accessed using The Plant List (https://www.theplantlist.org/), The International Plant Name Index Collaborators (https://www.gbif.org/), Plant of the World Online (https://www.plantsoftheworldonline.org/), and cross-referenced with Flora Malesiana series 1 Spermatophyta, Moraceae: Ficeae (Berg and Corner, 2005) to obtain the accepted name. The figs' conservation statuses were assessed via the International Union of Conservation of Nature (IUCN) Red List of Threatened Species $^{\mathrm{TM}}$ (https://iucnredlist.org/) and then compared with Indonesia's Ministry of Environment and Forestry regulation (Peraturan Menteri Lingkungan Hidup dan Kehutanan Republik Indonesia Nomor P.106/Menlhk/Setjen/Kum.1/12/2018 tentang Perubahan Kedua Atas Peraturan Menteri Lingkungan Hidup dan Kehutanan Nomor P.20/Menlhk/Setjen/Kum.1/6/2018 tentang Jenis Tumbuhan dan Satwa yang Dilindungi) to assess their national conservation statuses.

\section{RESULTS AND DISCUSSION}

\section{Figs diversity in Gianyar, Bali}

The fig (Ficus) is the largest genus in the Moraceae family with a more complex classification. Taxonomically, the genus of Ficus has three infrageneric classifications: subgenus, section, and subsection. In this study, 23 species composed of six subgenera were obtained (Table 1) with some documentation shown in Figure 2. The subgenus Urostigma was the most highly represented, with nine species, whereas the others were five species in the subgenus Sycidium, five species in the subgenus Sycomorus, two species in the subgenus Synoecia, and one species each in the subgenera Pharmacosycea and Ficus. Table 1 is also comprised of the habitus and fruit of the fig. Terrestrial trees or shrubs were the dominant habitus, followed by hemi-epiphytes and creeping figs. Some species could have more than one habitus, such as Ficus elastica and $F$. Superba, which are commonly found as trees, but could also appear as hemi-epiphytes. Fruit characteristics were added to provide an overview of potential frugivores. Large fruits, pale colors (usually greenish when mature), and cauliflorous/ramiflorous or flagelliflorous characteristics were commonly preferred by volant mammals, whereas fruits with bright color (contrasted with leaves) and placed axillarily or at the tips of branches were preferred by birds (Thornton et al. 1996). Among the 23 figs in Gianyar, 17 species had axillary figs, three species had cauliflorous/ramiflorous figs, two species had short flagelliflorous figs, and one species had long flagelliflorous figs. The IUCN Red List (2020) was accessed to derive the global conservation status of the figs. All fig species in this study were classified as Least Concern (LC), but categorized as an object of conservation ex-situ. 


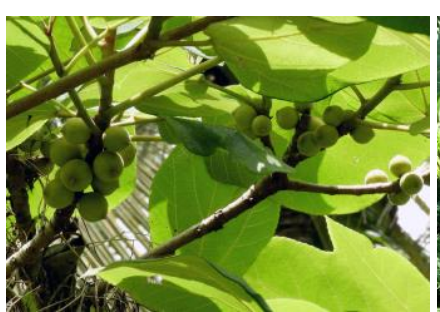

A

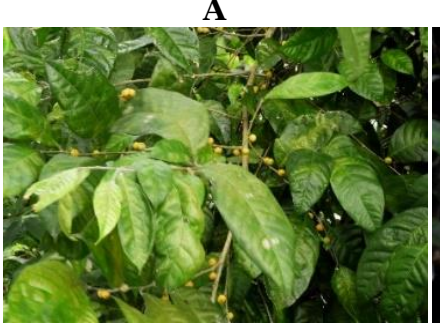

E

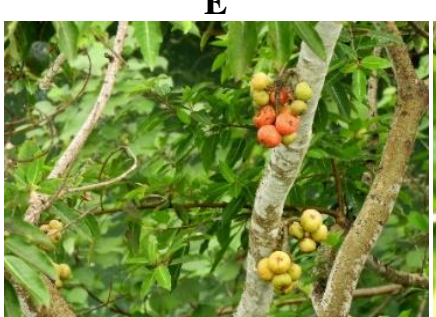

I

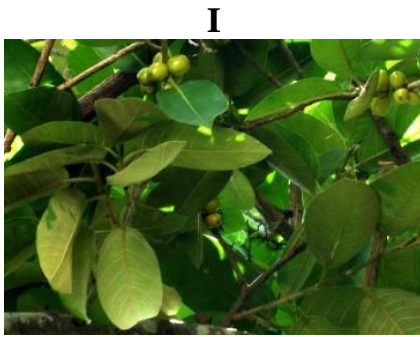

$\mathbf{M}$

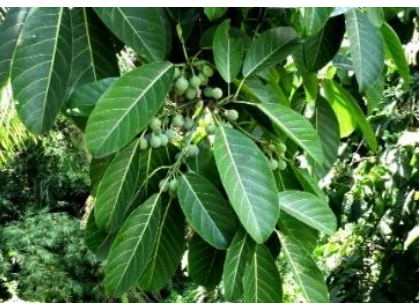

B

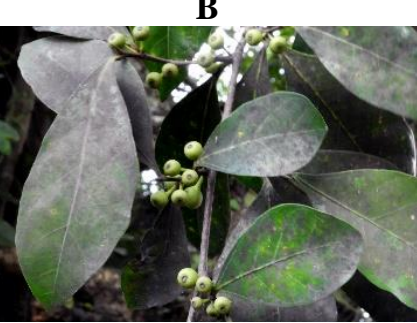

F

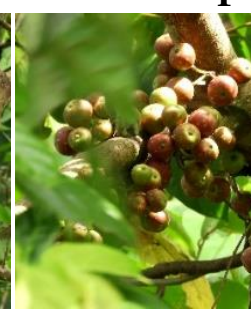

J

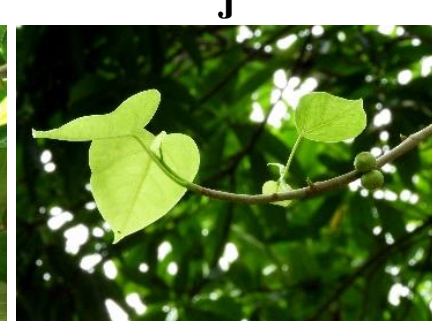

$\mathbf{N}$

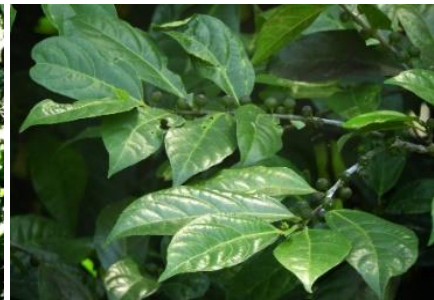

C

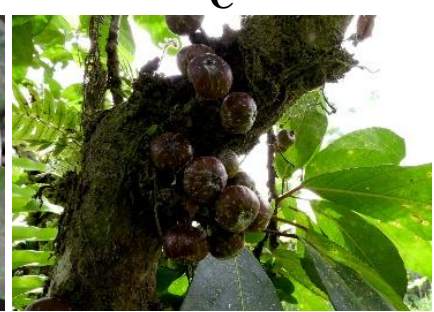

G

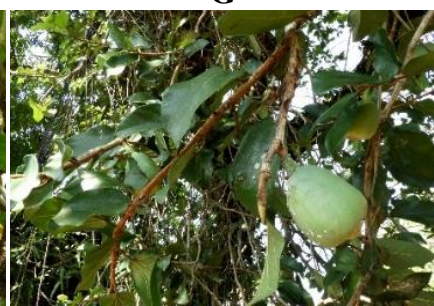

K

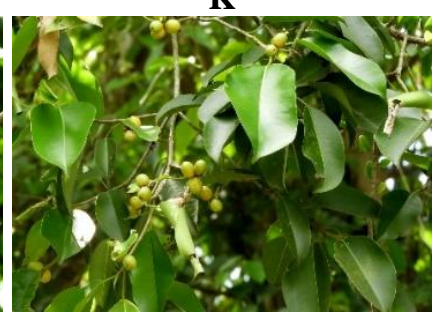

O

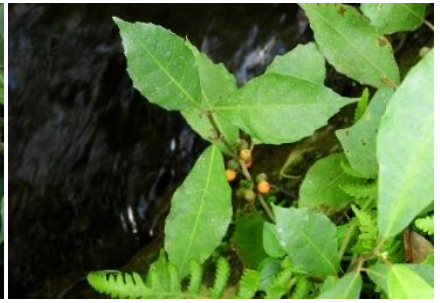

D

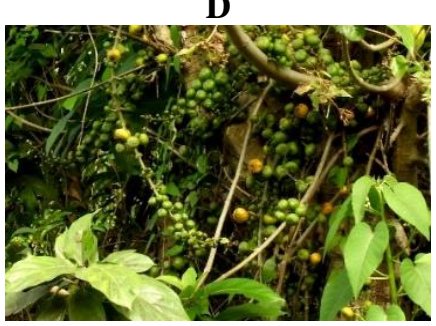

H

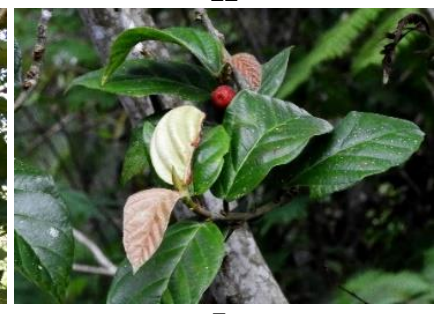

L

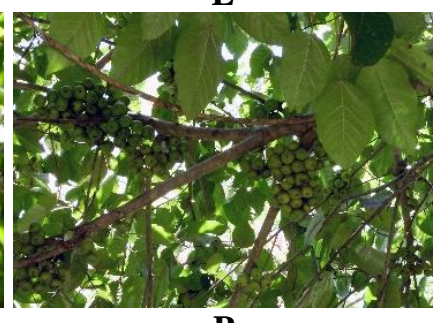

$\mathbf{P}$

Figure 2. Fig species found in Gianyar, Bali. A. Ficus fulva; B. Ficus callosa; C. Ficus ampelas; D. Ficus montana; E. Ficus subulata; F. Ficus tinctoria ssp. gibbosa; G. Ficus fistulosa brownish-purple fig variation; H. Ficus hispida; I. Ficus racemosa; J. Ficus variegata; K. Ficus pumila; L. Ficus trichocarpa; M. Ficus drupacea; N. Ficus rumphii; O. Ficus subcordata; and P. Ficus superba

Table 1. List of figs (Ficus: Moraceae) in Gianyar, Bali, Indonesia. The scientific names were derived from Berg \& Corner (2005) and https://www.plantsoftheworldonline.org/ with synonyms

\begin{tabular}{|c|c|c|c|}
\hline Scientific name & Common names & Habitus & Fruit \\
\hline $\begin{array}{l}\text { Subgenus Ficus } \\
\text { Ficus fulva Reinw. ex Blume } \\
\text { Synonyms: } \\
\text { Ficus apiculata Miq. } \\
\text { Ficus chlorocarpa Miq. } \\
\text { Ficus chrysocarpa } \text { Reinw. ex Blume } \\
\text { Ficus discolor Miq. } \\
\text { Ficus patens } \text { Ridl. } \\
\text { Ficus reinwardtii } \text { Link \& Otto } \\
\text { Ficus suborbicularis Miq. } \\
\text { Pogonotrophe flavidula Miq. }\end{array}$ & $\begin{array}{l}\text { Stinging fig } \\
\text { (English); hamerang } \\
\text { badak, kuyang, kebeg } \\
\text { (Indonesia); - (Bali) }\end{array}$ & Terrestrial tree. & $\begin{array}{l}\text { Pale green with hairy } \\
\text { surface; Axillary, } \\
\text { solitary or in pairs. }\end{array}$ \\
\hline $\begin{array}{l}\text { Subgenus Pharmacosycea } \\
\text { Ficus callosa Willd. } \\
\text { Synonyms: } \\
\text { Ficus basidentula Miq. } \\
\text { Ficus cinerascens Thwaites } \\
\text { Ficus cordatifolia Elmer } \\
\text { Ficus longespathulata Sata }\end{array}$ & $\begin{array}{l}\text { Calloused fig } \\
\text { (English); ilat-ilatan, } \\
\text { lasi (Indonesia); - } \\
\text { (Bali) }\end{array}$ & Terrestrial tree. & $\begin{array}{l}\text { Pale green with } \\
\text { pubescent surface; } \\
\text { Axillary, solitary or in } \\
\text { pairs. }\end{array}$ \\
\hline
\end{tabular}


Ficus malunuensis Warb.

Ficus porteana Regel

Ficus scleroptera Miq.

\section{Subgenus Sycidium}

Ficus ampelas Burm.f.
Synonyms:
Ficus asperior Miq.
Ficus bandana Miq.
Ficus guyeri Elmer

Ficus gul Lauterb. \& K. Schum

Synonyms:

Ficus bismarckiana Diels

Ficus griseifolia Corner

Ficus keyensis K.Schum. \& Lauterb.

Ficus manilensis Warb.

Ficus rudis Miq.

Ficus subconcolor Diels

Ficus montana Burm.f.

Synonyms:

Ficus ampullacea Wight ex Miq.

Ficus humilis Roxb.

Ficus inconstans Miq.

Ficus javanensis Dum.Cours.

Ficus loddigesii Heynh.

Ficus madurensis Miq.

Ficus purpurascens Desf.

Ficus purpurascens Blume

Ficus sclerocoma Miq.

Ficus smaragdina S.Moore

Ficus subulata Blume

Synonyms:

Ficus acuminata Roxb.

Ficus ancolana Miq.

Ficus confusa Elmer

Ficus dicarpa Blanco

Ficus driveri Elmer

Ficus erythropareia K.Schum. ex Warb.

Ficus iteoides Miq.

Ficus klinkii K.Schum. \& Lauterb.

Ficus salicifolia Miq.

Ficus sanhday Gagnep.

Ficus saxatilis Blume

Ficus sikkimensis Miq.

Ficus tinctoria ssp. gibbosa (Blume) Corner Synonyms:

Ficus gibbosa Blume

Ficus parasitica J.Koenig ex Willd.

Ficus angulata Miq.

\section{Subgenus Sycomorus}

Ficus fistulosa Reinw. ex Blume

Synonyms:

Ficus condensa King

Ficus curranii Merr.

Ficus grandidens Merr.

Ficus harlandii Benth.

Ficus lucbanensis Elmer

Ficus millingtonifolia Griff.

Ficus polysyce Ridl.

Ficus repandifolia Elmer

Ficus rubrovenia Merr.
Rough-leaf fig (English); amplasamplas, rampelas, hampelas (Indonesia); amplas-amplas (Bali)

Oakleaf fig (English); uyahan, amis mata (Indonesia); uyahuyah (Bali)

Dye fig, humped fig (English); kresek, kroya (Bali)

Yellow-stem fig (English); beunying (Indonesia); dadem (Bali)
Terrestrial tree or shrub.

Terrestrial tree or shrub.

Terrestrial small shrub with lack of branches.

Terrestrial climber or creeping fig. Could form dense branches at the base.
Hemiepiphyte or strangler fig which slowly replaced its host.

Terrestrial tree.
Green, gradually turn orange/yellow at maturity; Axillary, solitary.

Green; Cauliflorous.

Green, gradually turn red at maturity; Axillary with $1-5$ figs.

\section{Orange; Axillary,} solitary or in pairs.
Green, gradually turn orange or reddish at maturity; Axillary, solitary figs which clustered at the tip of branches.

Green, pale green, or brownish-purple; Cauliflorous or axillary at the leafy twigs. 


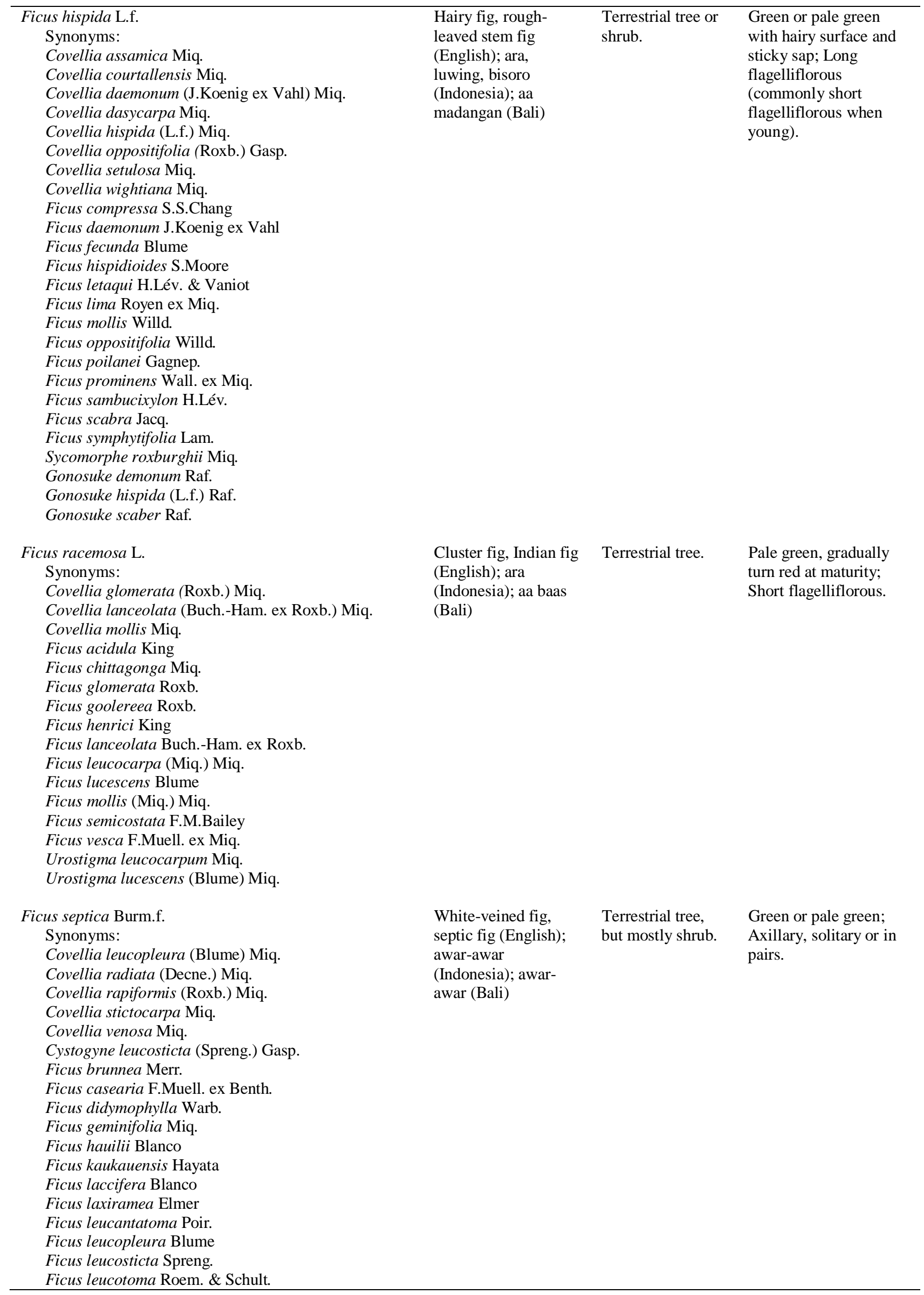


Ficus linearis Merr.

Ficus oldhamii Hance

Ficus paludosa Perr.

Ficus philippense Hérincq

Ficus radiata Decne.

Ficus rapiformis Roxb.

Ficus stictocarpa (Miq.) Miq.

Ficus verrucosa Vahl

Ficus variegata Blume

Synonyms:

Ficus agusanensis Elmer

Ficus amboinensis Kostel.

Ficus cerifera Blume

Ficus ceriflua Jungh.

Ficus chlorocarpa Benth.

Ficus compressitora Elmer

Ficus cordifolia Blume

Ficus domestica Zipp. ex Miq.

Ficus ehretioides F.Muell. ex Benth.

Ficus garciae Elmer

Ficus glochidiifolia Hayata

Ficus gummiflua Miq.

Ficus ilangoides Elmer

Ficus integrifolia Elmer

Ficus konishii Hayata

Ficus latsonii Elmer

Ficus paucinervia Merr.

Ficus subopaca Miq.

Ficus subracemosa Blume

Ficus sum Gagnep.

Ficus sycomoroides Miq.

Ficus tenimbrensis S.Moore

Ficus viridicarpa Corner

Sycomorus gummiflua Miq.

Urostigma cordifolium (Blume) Gasp.

Urostigma javanicum Miq.

\section{Subgenus Synoecia}

Ficus pumila L.

Synonyms:

Ficus hanceana Maxim.

Ficus longipedicellata H.Perrier

Ficus scandens Lam.

Ficus stipulata Thunb.

Ficus stipulata Lem.

Ficus vestita Desf.

Tenorea heterophylla Gasp.

Urostigma scandens Liebm.

Varinga repens Raf.

Plagiostigma pumila (L.) Zucc.

Plagiostigma stipulata Zucc.

Ficus trichocarpa Blume

Synonyms:

Ficus ahernii Merr.

Ficus filiformis Blume

Ficus nodulosa Zipp. ex Miq.

Ficus obtusa Hassk.

Ficus phaeopoda (Miq.) Miq.

Ficus piperifolia (Miq.) Miq.

Ficus platycaula Miq.

Ficus tawaensis Merr.

Pogonotrophe bornensis Miq.

Pogonotrophe javana Miq.

Pogonotrophe phaeopoda Miq.

Pogonotrophe piperifolia Miq.

Pogonotrophe pyrrhopoda Miq. Urostigma trichocarpum Miq.
Red-stem fig; greenfruited fig; variegated fig (English); ara,

nyawai, labo

(Indonesia); aa (Bali)
Terrestrial tree.

Pale green, gradually turn red at maturity; Short flagelliflorous.
Creeping fig,

climbing fig

(English); dolar

rambat (Indonesia); dolar mini (Bali)
Epiphyte.

Green or pale green, gradually turn purple at maturity; Axillary, solitary.
Climber or creeping fig.
Green, gradually turn red at maturity; Axillary, solitary. 


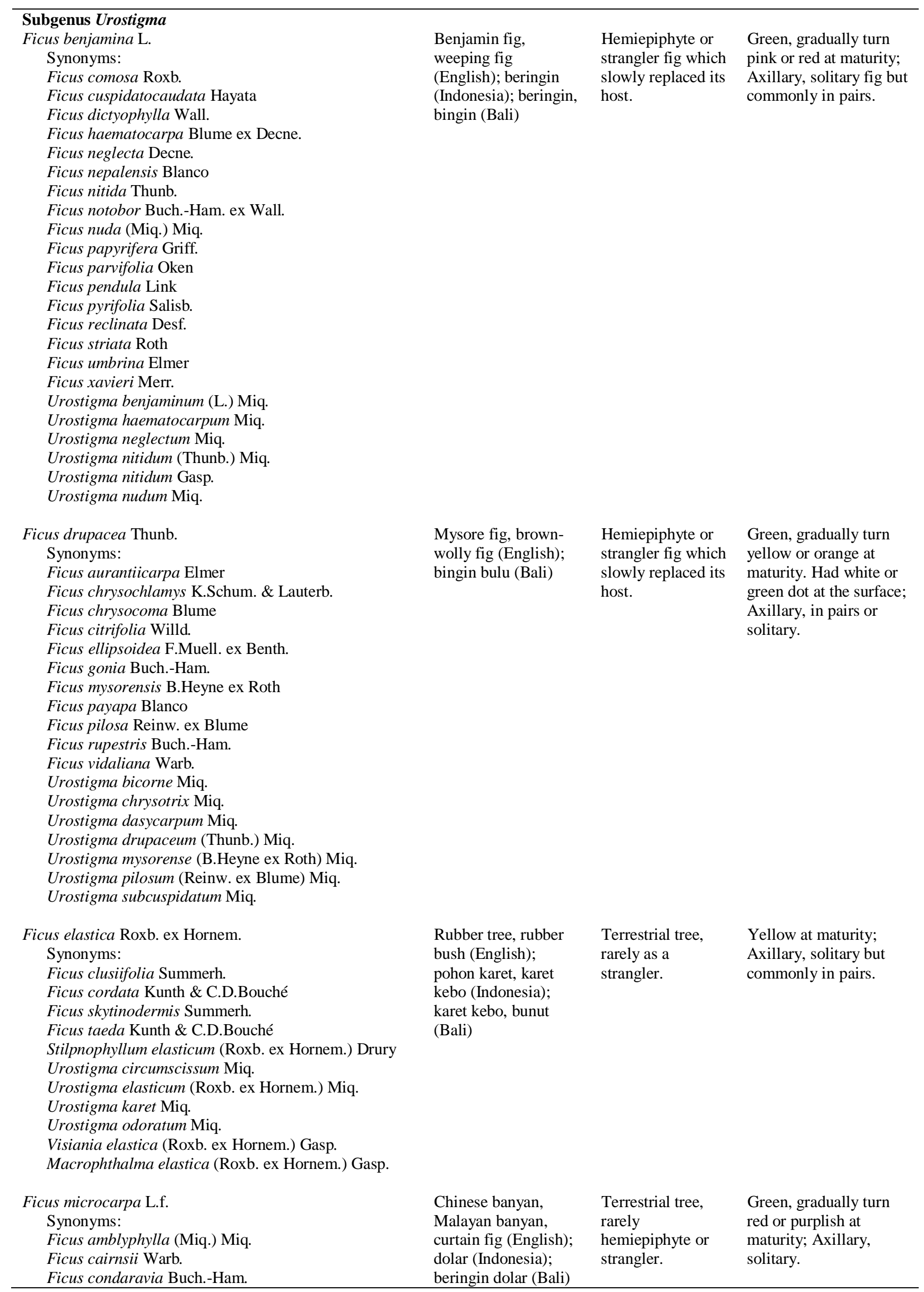


Ficus dahlii K.Schum.

Ficus dilatata Miq.

Ficus dyctiophleba F.Muell. ex Miq.

Ficus littoralis Blume

Ficus naumannii Engl.

Ficus regnans Diels

Ficus retusiformis H.Lév.

Ficus rubra Roth

Ficus thynneana F.M.Bailey

Urostigma amblyphyllum Miq.

Urostigma littorale (Blume) Miq.

Urostigma microcarpum (L.f.) Miq.

Urostigma pisiferum Miq.

Ficus religiosa $\mathrm{L}$.

Synonyms:

Ficus caudata Stokes

Ficus peepul Griff.

Ficus rhynchophylla Steud.

Ficus superstitiosa Link

Urostigma affine Miq.

Urostigma religiosum (L.) Gasp.

Ficus rumphii Blume

Synonyms:

Ficus affinior Griff.

Ficus conciliorum Oken

Ficus cordifolia Roxb.

Ficus damit Gagnep.

Ficus populiformis Schott ex Miq.

Ficus populnea Kunth \& C.D.Bouché

Urostigma cordifolium Miq.

Urostigma rumphii (Blume) Miq.

Ficus subcordata Blume

Synonyms:

Ficus acrorrhyncha Summerh.

Ficus balica (Miq.) Boerl.

Ficus calophylloides Elmer

Ficus fairchildii Backer

Ficus garciniifolia Miq.

Ficus polygramma Corner

Urostigma balicum Miq.

Urostigma subcordatum Miq.

Ficus superba (Miq.) Miq.

Synonyms:

Ficus petiolata Reinw. ex Miq.

Ficus tenuipes S.Moore

Ficus timorensis Decne.

Urostigma accedens Miq.

Urostigma superbum Miq.

Ficus virens Aiton

Synonyms:

Ficus ampla Kunth \& C.D.Bouché

Ficus carolinensis Warb.

Ficus cunninghamii (Miq.) Miq.

Ficus fraseri (Miq.) F.Muell.

Ficus glabella Blume

Ficus infrafoliacea Buch.-Ham. ex Sm.

Ficus lambertiana (Miq.) Miq.

Ficus monticola Miq.

Ficus nesophila (Miq.) F.Muell.

Ficus nitentifolia S.Moore

Ficus psychotriifolia (Miq.) Miq.

Ficus racemosa Willd.

Ficus scandens Buch.-Ham.

Ficus terminalioides Griff.

Ficus terminalis B.Heyne ex Roth
Bodhi, sacred fig, $\quad$ Terrestrial tree. peepul tree (English);

Bodhi (Indonesia);

Bodhi (Bali)

Rumph's fig

(English); ancak

(Bali)

Decidous fig, sea fig

(English); krasak,

kresek (Indonesia);

kresek (Bali)

White fig, grey fig (English); ampulu (Indonesia)
Terrestrial tree, rarely hemiepiphyte or strangler.

Terrestrial tree, rarely

hemiepiphyte or strangler.

Terrestrial tree, rarely hemiepiphyte or strangler.

Hemiepiphyte or strangler fig which slowly replaced its host.
Greenish yellow, gradually turn red or purplish at maturity; Axillary, solitary but commonly in pairs.

Pinkish, gradually turn purple or black at maturity; Axillary, solitary but commonly in pairs and densely at the tip of branches.

Green or pale green, gradually turn yellow or orange at maturity; Axillary, solitary or in pairs.

Green or pale green; Cauliflorous.

Pinkish white; Axillary, solitary. 


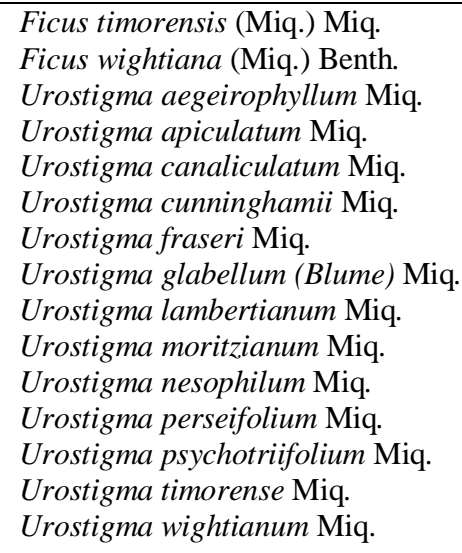

Table 2. Distribution of figs (Ficus: Moraceae) in Gianyar, Bali. Note: I: Blahbatuh; II: Gianyar; III: Sukawati; IV: Ubud; V: Payangan; VI: Tampaksiring; VII: Tegallalang

\begin{tabular}{|c|c|c|c|c|c|c|c|}
\hline \multirow{2}{*}{ Species } & \multicolumn{7}{|c|}{ Distribution Area } \\
\hline & I & II & III & IV & $\mathbf{V}$ & VI & VII \\
\hline Ficus fulva Reinw. ex Blume & & & & & & + & \\
\hline Ficus callosa Willd. & & & & + & & + & \\
\hline Ficus ampelas Burm.f. & & + & & + & + & + & \\
\hline Ficus gul Lauterb. \& K. Schum & & + & + & & & & \\
\hline Ficus montana Burm.f. & + & + & + & + & + & + & + \\
\hline Ficus subulata Blume & + & + & & & + & + & + \\
\hline Ficus tinctoria ssp. gibbosa (Blume) Corner & & + & & & & & \\
\hline Ficus fistulosa Reinw. ex Blume & & + & & & + & + & + \\
\hline Ficus hispida L.f. & + & + & + & + & + & + & + \\
\hline Ficus racemosa $\mathrm{L}$. & & + & + & + & + & & + \\
\hline Ficus septica Burm.f. & + & + & + & + & + & + & + \\
\hline Ficus variegata Blume & + & + & + & + & + & & + \\
\hline Ficus pumila $\mathrm{L}$. & + & + & + & + & + & + & + \\
\hline Ficus trichocarpa Blume & & + & & & & + & + \\
\hline Ficus benjamina $\mathrm{L}$. & + & + & + & + & + & + & + \\
\hline Ficus drupacea Thurnb. & & + & & & & + & + \\
\hline Ficus elastica Roxb. ex Hornem. & + & & & + & & & \\
\hline Ficus microcarpa L.f. & & + & & + & & & \\
\hline Ficus religiosa $\mathrm{L}$. & & + & + & + & & & \\
\hline Ficus rumphii Blume & & & + & + & & + & \\
\hline Ficus subcordata Blume & & & & & & & + \\
\hline Ficus superba (Miq.) Miq. & + & + & + & + & + & + & + \\
\hline Ficus virens Aiton & & + & & & & & \\
\hline Total & 9 & 18 & 11 & 14 & 11 & 14 & 13 \\
\hline
\end{tabular}

\section{Fig distribution in Gianyar, Bali}

Fig distribution in Gianyar is presented in Table 2. Area II (Gianyar) had the highest number of fig species obtained, 18 species, whereas Area I (Blahbatuh) had the lowest number, nine species. Naturally, fig distribution is related to the dispersal agent and environment. Ficus members are commonly found growing around a body of water, except for strangler figs and $F$. Pumila which are used as ornamental plants. During exploration, some species were rarer than others, such as $F$. fulva which only found in Tampaksiring, $F$. tinctoria ssp. gibbosa and $F$. virens in Gianyar, and $F$. subcordata in the Tegallalang areas. In contrast, some species (F. montana, F. hispida, F. septica, $F$. pumila, $F$. benjamina, and $F$. superba) were found in all the locations studied.

\section{Discussion}

Figs (Ficus) are pantropical plants consisting of approximately consist of 735 species, 367 of which are distributed in the Asia-Australasian region (Berg and Corner 2005). Indonesia has among the highest fig diversity, with Borneo as its center of diversity. Berg and Corner (2005) reported 138-141 species in Borneo, 94-97 species in Sumatera, 79-81 species in Moluccas (Maluku), 78-81 species in Celebes (Sulawesi), 74-77 species in Java, and 34 species on Lesser Sunda Island (Bali and Nusa Tenggara). Ficus is also known as a highly dispersed genus because of its extreme range of growth forms, thus making it more adaptable in various types of ecosystems. This genus could be found as trees, shrubs, climbers and scramblers, epiphytes, or hemi-epiphytes (stranglers).

Ficus is composed of 6 subgenera: Urostigma, Pharmacosycea, Ficus, Synoecia, Sycidium, and Sycomorus. On Lesser Sunda Island, Urostigma was the subgenus with the highest diversity relative to the others (Berg \& Corner 2005), which correlated with this research. Berg and Corner (2005) found that of the 34 species on Lesser Sunda Island, 12 species were from the subgenus Urostigma, four species from Pharmacosycea, one species from Ficus, three species from Synoecia, eight species from Sycidium, and six species from Sycomorus. Urostigma is also a subgenus with many hemi-epiphyte species. Both large and small hemi-epiphyte Urostigma figs attract many frugivorous birds and mammals (Sreekar et al. 2010). Hemi-epiphyte figs do not depend on water resources or water bodies because of their adaptability to dehydration, however, they depend on their host tree (Pothasin et al. 2014). Because these hemi-epiphytes need large trees as proper hosts, logging could affect the diversity of hemiepiphytes, sequentially impacting the frugivore diversity (Harrison et al. 2003).

All species in the subgenus Urostigma were monoecious (Berg and Corner 2005; Cruaud et al. 2012) with a wide variety of pollinating insects (Cruaud et al. 2012). The monoecious-dioecious traits in figs were the results of reciprocal evolution in mutualistic fig/figpollinating wasp interactions that affected the diversity of figs (Cruaud et al. 2012; Zhang et al. 2019). A high variety of pollinating insects made monoecious figs occupy more areas than dioecious figs, but in terms of local abundance, 
dioecious figs are more abundant (Harrison 2003; Dev et al. 2011). Complex interactions between insects and monoecious figs in Urostigma affect the morphological character divergence. Chantarasuwan et al. (2015) used a molecular approach to solve the taxonomic uncertainties within Urostigma because of its high morphological variability and exhibit overlapping distribution.

Not only were all the Urostigma species in this study monoecious, they were also all strangler figs: $F$. benjamina, $F$. drupacea, $F$. elastica, $F$. microcarpa, $F$. religiosa, $F$. rumphii, $F$. subcordata, $F$. superba, and $F$. virens. Monoecious strangler figs have wider geographical distribution (Harrison et al. 2003; Chantarasuwan et al. 2015) caused by animal dispersal and human introduction. Ficus benjamina, F. drupacea, F. elastica, F. microcarpa, and $F$. religiosa are Asian figs that were heavily introduced to other countries as cultivated plants (Berg and Corner 2005). In the view of Hindu culture in Bali, figs are also an integrated part of religion. The banyan tree ( $F$. benjamina $)$ plays the main part in the ceremony of the ngangget don bingin phase, which is one of the phases in the cremation ceremony or ngaben (Figure 3.A-B). That is why at least one banyan tree is always found in every village. The banyan tree is also considered a plant with a mystical ambiance and is associated with fertility; thus, its existence has been venerated in many traditional cultures (Harrison et al. 2003). This is also the case for $F$. religiosa, which is globally known as the bodhi or holy fig. Ficus religiosa is mainly found at the temple (pura) or vihara, and is believed to give a serene ambiance. Although this fig is popular in Malesia, its native distribution in Asia did not reach the Malesian region without human introduction (Berg and Corner 2005).

The culturally associated banyan tree in Bali indirectly supports the ecosystem. Shanahan et al. (2001) compiled data on frugivores and found out that the banyan tree $(F$. benjamina) can support 84 species of frugivores. Other strangler fig species also supported remarkable numbers of frugivores. Ficus virens could support 90 species of frugivores, and $F$. drupacea up to 63 species of frugivores
(Shanahan et al. 2001). The number of frugivores for $F$. drupacea might vary due to its intraspecific variations. Ficus drupacea had two major variations (forms) based on its biogeographical distribution: the stipitate receptacles form, which is found in eastern New Guinea, and the hairy (twigs, petioles, and laminas) form, which is found in the western New Guinea (Berg and Corner 2005). Corresponding with the prior research, the hairy form of $F$. drupacea was found in this study. There was also a variation in its fruit surface. Some fruit could have a glabrous surface, whereas others could be coarser. This variation might be correlated with fig/fig-pollinating wasp interaction. Wang et al. (2013) hypothesized that the pollinator specificity in figs could be a result of a combination of the scent and surface texture of the fruit to prevent misleading pollinators.

The five species of the subgenus Sycidium in this study were $F$. ampelas, $F$. gul, F. montana, $F$. subulata, and $F$. tinctoria. The species in this subgenus are relatively common around the world, mainly distributed on riverbanks and in humid areas, and a few species are found on the beaches or in grasslands (Berg and Corner 2005). All Sycidium species are dioecious. Dioecious figs are more abundant than monoecious figs in tropical regions, usually in the forms of small trees, shrubs, or climbers (Bain et al. 2014). Ficus ampelas is known as amplasamplas because of its rough leaves. This species is widespread in Indonesia except for Borneo (Berg and Corner 2005). Ficus tinctoria and F. subulata also have a wide distribution in Indonesia. Ficus tinctoria has two subspecies: F. tinctoria ssp. tinctoria, which is distributed in eastern Bali, and F. tinctoria ssp. gibbosa, which is distributed in western Bali (Berg and Corner 2005). Some people in Bali distinguish $F$. tinctoria from $F$. benjamina by its leaves. The leaf vein in F. tinctoria is more obvious than in $F$. benjamina. This is necessary because those two species could appear on the same host tree, such as in Gianyar, where an Alstonia scholaris tree is strangled with $F$. benjamina and F. tinctoria.

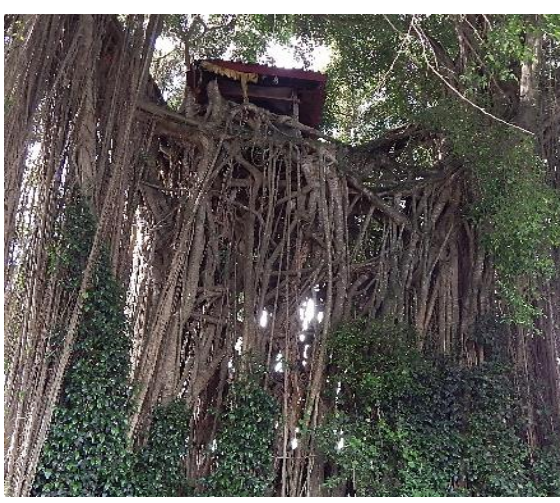

A

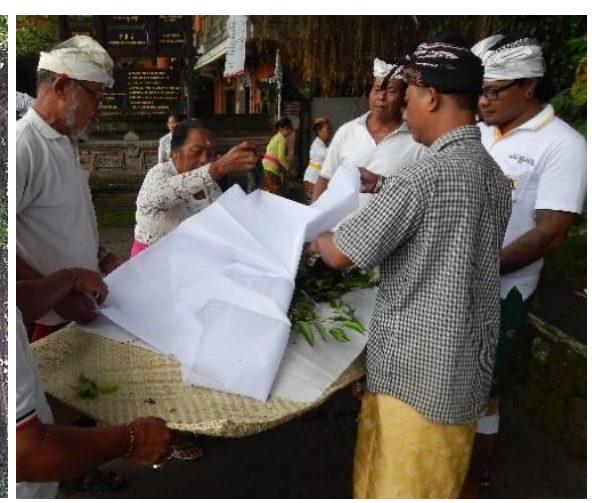

B

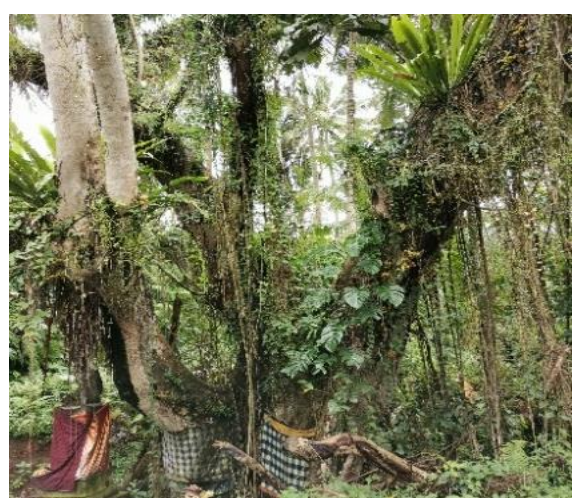

C

Figure 3. Traditional culture is associated with figs. A. Ficus benjamina in Ubud with massive roots; B. Ngangget don bingin phase in the cremation ceremony sequence showing people collecting $F$. benjamina leaves using a particular tool; C. The culturally protected $F$. racemosa at Tegallalang supports many forms of plant and animal life 
Ficus subulata is a climber fig that shares a unique interaction with its host. The lower parts of the plant, usually in a shaded area, form small canopy by formation of its dense branches below the host's canopy. The canopy of $F$. subulata could act as protection for frugivores or create a specific niche for ground animals. Some of the branches climb the host tree with loose branches until reaching a suitable height. Both the upper and lower parts can produce a massive amount of axillary fruit. Even though its mature stage is similar to hemi-epiphytes, a molecular study showed that climber figs developed from a shrub form (Zhang et al. 2020).

Ficus montana is a small shrub fig commonly found in pastureland, rice fields, or abandoned areas. This species is very adaptable to unfavorable and unpredictable conditions (Bain et al. 2014) and is mostly recognized as a weed. In Bali and Java, it is known as uyah-uyah or uyahan and used as food for ruminants. Ficus montana has varied leaf morphology in terms of shape (oval, obovate, oblong), margin (entire, sparsely dentate, serrated, lobed as oak leaf), apex (acute, acuminate, rarely caudate). Generally, the leaf color is green, except for the $F$. montana var. purpurascens, which is purple on the underside of the leaf (Tarachai et al. 2012). The amount of fruit (syconia) is also variable, usually 1-2, but sometimes more than five. Ficus montana is often used for studying the relationship between the fig and its pollinator (Kradibia tentacularis: Aganoidae). Suleman et al. (2015) reported interaction between $F$. montana and its pollinator while studying the floral ratio of active and passive pollination.

The subgenus Sycomorus consists of tree, small tree, and shrub figs, such as $F$. hispida, $F$. septica, $F$. racemosa, $F$. variegata, and F. fistulosa. Most Sycomorus species are dioecious. Dioecious figs produce massive fruit all year round (Bain et al. 2014), but they have short-range pollinators because their fruits grow under the canopy (Harrison 2003; Harrison and Rasplus 2006). This could increase the local extinction risk due to pollinator disappearance (Harrison and Rasplus 2006). Some species are fast-growing figs with quickly maturing fruits and are common in disturbed areas, such as $F$. septica and $F$. hispida. Ficus is a notable genus for forest restoration in disturbed landscapes (Cottee-Jones et al. 2016), and these two species appeared at the early stage of succession on Krakatau Island as pioneer trees or pioneer figs (Thornton et al., 1996). Although both species were adaptable in various types of habitats, those species have different colonization strategies. Ficus septica tends to produce fruit as soon as possible. It is common to see $1.5 \mathrm{~m} \mathrm{~F}$. septica plants producing fruit. In contrast, $F$. hispida tends to produce fruit only under certain conditions, but in massive quantities. For example, a $4 \mathrm{~m} \mathrm{~F}$. hispida plant could produce many fruits in short and long flagelliflorous forms (Figure 2.H). In forest restoration, F. hispida is an excellently ranked species (Kuaraksa et al. 2012).

Ficus fistulosa is locally known as dadem. In Tegallalang, young leaves of the F. fistulosa could be processed as vegetables or used as a forage plant for ruminants. This species is abundant at the roadside in
Tegallalang, Tampaksiring, and Payangan, with a height < $3 \mathrm{~m}$ because it is regularly trimmed. There are two variations of $F$. fistulosa based on its fruit color, green (green to greenish-yellow when mature) and brownish-red (red to brownish-red when mature). The brownish-red fruit is rarer than the green; at lower altitudes (below $300 \mathrm{~m}$ ASL), only green fruit was found. Ficus fistulosa also bears fruit in axillary and cauliflorous form. The axillary fruits have a depressed-globose shape and are larger than the cauliflorous fruits, whereas the cauliflorous fruits are globose. The cauliflorous fruits only appear on adult trees, but the axillary fruits appear in both the juvenile and adult/mature stages of growth.

Some of the figs are widespread species that were found in many geographical areas, such as $F$. racemosa and $F$. variegata (Berg and Corner 2005). These species can grow up to $20 \mathrm{~m}$ high or more and are characterized by producing contrast red fruit. Among the mature red fruit, groups of pale green or yellow fruit were also found. The pale green group, known as gall fruit, are believed to have a correlation with primates' vision evolution (Berg and Corner 2005). Ficus variegata has variations in fruiting times between male and female plants. Male $F$. variegata trees are semi-deciduous with 4-month fruiting periods, whereas the female trees are deciduous with fruiting periods throughout the year (Kuaraksa et al. 2012). Even though $F$. variegata and $F$. racemosa have similar habitus types, $F$. racemosa has its own value for elderly people in Gianyar. When there was a large eruption of Mount Agung in 1963, people in Gianyar lacked a food source and started to consume the fruit of $F$. racemosa as a substitute for rice. In some areas in Bali, $F$. racemosa is also protected because of a belief that the plant has contributed to protecting water sources. Soejono et al. (2013) stated that the fig tree is a perfect plant for restoration and water source protection because of its broad and deep root system that increases water infiltration and its dense and wide canopy, which can reduce the speed of raindrops. The dense branching in $F$. racemosa that supports many epiphyte plants (Figure 3.C) also contributing in the reduction of the speed of raindrops.

Ficus pumila and F. trichocarpa are classified in the subgenus Synoecia, which is dominated by climbing figs (Zang et al. 2020). Ficus pumila is an introduced species that is frequently cultivated in Indonesia as an ornamental plant (Backer and van den Brink 1965; Berg and Corner 2005), especially as a wall-creeping plant. In Bali, the fruiting phase of $F$. pumila is rare because periodically trimming the plant affects its growth and development. The fruiting $F$. pumila is commonly found in neglected places, where it grows uncontrollably, climbs the nearest tree, and then produces many fruits. Ficus pumila is not only used for ornamental plant, but also has special taxonomic characteristics. The taxonomic position of the cultivated $F$. pumila excites many discussions regarding the possibility of interspecific hybridization between $F$. pumila and $F$. deltoidea (Zhang et al. 2020) or between $F$. pumila and $F$. thunbergii (Tsai et al. 2015). 
As opposed to $F$. pumila, $F$. trichocarpa is not an ornamental plant. This plant is usually found creeping on other tall trees near a body of water, such as a river, or in a foggy area. The polymorphism of $F$. trichocarpa leaves can be separated into bathyphylls and acrophylls (Berg and Corner 2005). Bathyphylls are sterile leafy twigs that creep on the stem host, have smaller leaves, and have many hairs, whereas acrophylls are fertile twigs that form fruits and have larger leaves. The leaf shape also varies. Bathyphylls of $F$. tricocarpha are commonly spear-shaped, oval, obovate, or oblong, and acrophylls are usually obovate and thicker.

Ficus callosa and F. fulva were the only species in the subgenera Pharmacosyceae and Ficus in this study. Both of those species are abundant on riverbanks. The fig is an important plant in riparian zones as a means of stream stabilization, flood reduction, shade for animals, and as a food source (Pothasin et al. 2014). Ficus callosa reach $>25$ $\mathrm{m}$ in height and produce small buttresses, whereas $F$. fulva does not reach more than $15 \mathrm{~m}$ in height. Seedlings of $F$. callosa are easy to find, dispersed along the water's edge to drier areas. Ficus callosa has leaf variations between the juvenile and adult plants. The juvenile leaf of $F$. callosa is oblong-lanceolate with a spinulose-dentate base, whereas the adult leaf is oval-shaped, which matches with the annotations of Berg and Corner (2005). Ficus fulva also has leaf variations. The shape of the juvenile $F$. fulva leaf is subpalmate with 3-5 lobes and turns oval, obovate, or pandurate when it matures.

In conclusion, 23 species of figs were found in Gianyar District and were identified and classified into six subgenera. The subgenus Urostigma had the highest species diversity with nine species, followed by Sycidium with five species, Sycomorus with five species, Synoecia with two species, Pharmacosycea with one species, and Ficus with one species. Some species in Urostigma and Sycomorus had strong relationships with Balinese culture, such as $F$. benjamina, $F$. rumphii, $F$. religiosa, and $F$. racemosa.

\section{ACKNOWLEDGEMENTS}

Thank you to The Institution of Research and Community Services, Udayana University, Denpasar, Indonesia for the research grant in the Penelitian Unggulan Program Studi scheme with the contract 023.17.2.677526/2020. We also thank the Faculty of Mathematics and Natural Sciences, Udayana University; the research team: Viryanando Evan Rahardja, Egmont Congdenjit, Komang Kartika Indi Swari, and Dewa Ayu Intan Tirta Sari for their supports and assistances; and also Prof. Purnomo, Dr. Wawan Sujarwo, Dr. Mahfut, and Dr. Nery Sofiyanti for the valuable feedback.

\section{REFERENCES}

Backer CA, van den Brink RCB. 1965. Flora of Java (Spermatophytes only) Volume II. N.V.P. Noordhoff-Groningen, Netherlands.
Bain A, Chou L-S, Tzeng H-Y, Ho Y-C, Ching Y-P, Chen W-H, Chio YT, Li G-Y, Yang H-W, Kjellberg F, Hossaert-McKey M. 2014. Plasticity and diversity of the phenology of dioecious Ficus species in Taiwan. Acta Oecologia 57: 124-134. DOI: 10.1016/j.actao.2013.10.004.

Berg CC, Corner EJH. 2005. Moraceae: Ficeae. Flora Malesiana - Series 1, Spermatophyta 17 (2): 1-702.

BPS-Statistics of Gianyar. 2019. Gianyar Regency in figures 2019. BPSStatistics of Gianyar, Gianyar.

Bravo A, Harms KE, Emmons LH. 2012. Keystone resource (Ficus) chemistry explains lick visitation by frugivorous bats. J Mammal 93 (4): 1099-1109.

Chantarasuwan B, Berg CC, Kjellberg F, Ronsted N, Garcia M, Baider C, van Welzen PC. 2015. A new classification of Ficus subsection Urostigma (Moraceae) based on four nuclear DNA markers (ITS, ETS, G3pdh, and ncpGS), morphology, and leaf anatomy. PLoS ONE 10 (6): e0128289. DOI: 10.1371/journal.pone.0128289.

Cottee-Jones HEW, Bajpai O, Chaudhary LB, Whittaker RJ. 2016. The importance of Ficus (Moraceae) trees for tropical forest restoration. Biotropica 48 (3): 413-419. DOI: 10.1111/btp.12304.

Cruaud A, Ronsted N, Chantarasuwan B, Chou LS, Clement WL, Couloux A, Cousins B, Genson G, Harrison RD, Hanson PE, Hossaert-Mckey M, Jabbour-Zahab R, Jousselin E, Kerdelhué C, Kjellberg F, Lopez-Vaamonde C, Peebles J, Peng YQ, Santinelo Pereira RA, Schramm T, Ubaidillah R, Van Noort S, Weiblen GD, Yang DR, Yodpinyanee A, Libeskind-Hadas R, Cook JM, Rasplus JY, Savolainen V. 2012. An Extreme case of plant-insect codiversification: Figs and fig-pollinating wasps. Syst Biol 61 (6): 1029-1047. DOI: 10.1093/sysbio/sys068.

Dev SA, Kjellberg F, Hossaert-McKey M, Borges RM. 2011. Fine-scale population genetic structure of two dioecious Indian keystone species, Ficus hispida and Ficus exasperata (Moraceae). Biotropica 43 (3): 309-316. DOI: 10.1111/j.1744-7429.2010.00704.x.

Hadisusanto S, Eprilurahman R, Purnomo, Yudha DS, Trijoko, Sulistyono, Sudibyo P, Asti HA, Ramadani RS, Sancayaningsih RP, Pranoto FXS, Muhtianda IA. 2018. Keanekaragaman hayati di Gianyar. UGM Press: Yogyakarta.

Harrison RD. 2003. Fig wasp dispersal and the stability of a keystone plant resource in Borneo. Proceedings of the Royal Society B: Biological Sciences 270: 76-79. DOI: 10.1098/rsbl.2003.0018

Harrison RD. 2005. Figs and the diversity of tropical rainforests. BioScience 55 (12): 1053-1064

Harrison RD, Hamid AA, Kenta T, Lafrankie J, Lee H Sen, Nagamasu H, Nakashizuka T, Palmiotto P. 2003. The diversity of hemi-epiphytic figs (Ficus; Moraceae) in a Bornean lowland rain forest. Biol J Linn Soc 78 (4): 439-455. DOI: 10.1046/j.0024-4066.2002.00205.x.

Harrison RD, Rasplus JY. 2006. Dispersal of fig pollinators in Asian tropical rain forests. J Trop Ecol 22 (6): 631-639. DOI: $10.1017 / \mathrm{S} 0266467406003488$.

Kuaraksa C, Elliott S, Hossaert-McKey M. 2012. The phenology of dioecious Ficus spp. tree species and its importance for forest restoration project. For Ecol Manag 265: 82-93. DOI: 10.1016/j.foreco.2011.10.022

Lim V-C, Clare EL, Littefair JE, Ramli R, Bhassu S, Wilson J-J. 2017. Impact of urbanisation and agriculture on the diet of fruit bats. Urban Ecosyst. DOI: 10.1007/s11252-017-0700-3.

Lok AFSL, Ang WF, Ng BYQ, Leong TM, Yeo CK, Tan HTW. 2013. Native fig species as a keystone resource for the Singapore urban environment. Raffles Museum of Biodiversity Research National University of Singapore: Singapore. DOI: 10.13140/2.1.1217.5687

Medeiros LP, Garcia G, Thompson JN, Guimarães PR. 2018. The geographic mosaic of coevolution in mutualistic networks. PNAS 115 (47): 12017-12022. DOI: 10.1073/pnas.1809088115.

Myers N, Mittermeier RA, Mittermeier CG, Fonseca GAB, Kent J. 2000. Biodiversity hotspots for conservation priorities. Nature 403 (2): 853858.

Peraturan Menteri Lingkungan Hidup dan Kehutanan Republik Indonesia Nomor P.106/Menlhk/Setjen/Kum.1/12/2018 tentang Perubahan Kedua Atas Peraturan Menteri Lingkungan Hidup dan Kehutanan Nomor P.20/Menlhk/Setjen/Kum.1/6/2018 tentang Jenis Tumbuhan dan Satwa yang Dilindungi. [Indonesian]

Plants of the World Online. 2020. https://plantsoftheworldonline.org/ on 2020-12-10.

Pothasin P, Compton SG, Wangpakapattanawong P. 2014. Riparian Ficus tree communities: The distribution and abundance of riparian fig trees 
in Northern Thailand. PLoS One 9 (10): e108945. DOI: 10.1371/journal.pone.0108945.

Rugayah, Retnowati A, Windadri FI, Hidayat A. 2004. Pengumpulan Data Taksonomi. In: Rugayah, Widjaya EA and Praptiwi (eds) Pedoman Pengumpulan Data Keanekaragaman Flora. Puslit Biologi, LIPI, Bogor. [Indonesian]

Shanahan M, Samson SO, Compton SG, Corlett R. 2001. Fig-eating by vertebrate frugivores: A global review. Biol. Rev. Camb. Philos. Soc. 76 (4): 529-572. DOI: 10.1017/S1464793101005760.

Soejono, Budiharta S, Arisoesilaningsih E. 2013. Proposing local trees diversity for rehabilitation of degraded lowland areas surrounding springs. Biodiversitas 14 (1): 37-42. DOI: 10.13057/biodiv/d140106.

Sreekar R, Le NTP, Harrison RD. 2010. Vertebrate assemblage at a fruiting fig (Ficus caulocarpa) in Maliau Basin, Malaysia. Trop. Conserv Sci 3 (2): 218-227. DOI: 10.1177/194008291000300208.

Sujarwo W, Caneva G, Zuccarello V. 2020. Patterns of plant use in religious offerings in Bali (Indonesia). Acta Bot Bras 34 (1): 40-53. DOI: $10.1590 / 0102-33062019 a b b 0110$

Suleman N, Quinnell RJ, Compton SC. 2014. Floral ratios in the figs of Ficus montana span the range from actively to passively pollinated fig trees. Acta Oecol. 57: 67-72. DOI: 10.1016/j.actao.2013.11.005.

Tarachai Y, Pothasin P, Tanming W, Compton SG. 2012. The distribution and ecology of the purple form of Ficus montana in western Thailand. Thai For Bull Bot 40: 26-30.

The International Plant Names Index Collaborators. 2019. International Plant Names Index. DOI: 10.15468/uhllmw

The Plant List. 2012. Accessed via http://www.theplantlist.org/1.1/browse/A/Moraceae/Ficus/ on 202009-30.

Thornton IWB, Compton SG, Wilson CN. 1996. The role of animals in the colonization of the Krakatau Islands by fig trees (Ficus species). J Biogeogr 23 (4): $577-592$. DOI: $10.1111 / \mathrm{j} .1365-$ 2699.1996.tb00019.x.
Tsai L, Hayakawa H, Fukuda T, Yokoyama J. 2015. A breakdown of obligate mutualism on a small island: An interspecific hybridization between closely related fig species (Ficus pumila and Ficus thunbergii) in Western Japan. Am J Plant Sci 6: 126-131. DOI: 10.4236/ajps.2015.61014.

van Steenis CGGJ. 1972. The Mountain Flora of Java. E.J. Brill, Leiden

Walther BA, Geier J, Chou L-S, Bain A. 2017. The figs of winter: Seasonal importance of fruiting fig trees (Ficus: Moraceae) for urban birds. Acta Oecologia. DOI: 10.1016/j.actao.2017.11.015.

Wandrag EM, Dunham AE, Duncan RP, Rogers HS. 2017. Seed dispersal increases local species richness and reduces spatial turnover of tropical tree seedlings. Proc Natl Acad Sci USA 114 (40): 1068910694. DOI: 10.1073/pnas.1709584114.

Wang G, Compton SG, Chen J. 2013. The mechanism of pollinator specificity between two sympatric fig varieties: A combination of olfactory signals and contact cues. Ann Bot. 111 (2): 173-181. DOI: $10.1093 / \mathrm{aob} / \mathrm{mcs} 250$.

Wang R-W, Yang C-Y, Zhao G-F, Yang J-X. 2005. Fragmentation effects on diversity of wasp community and its impact on fig/fig wasp interaction in Ficus racemosa L. J Integr Plant Biol 47 (1): 20-26

Womersley JS. 1981. Plant collecting and herbarium development. Food and Agriculture Organization of United Nations, Rome.

Xu L, Harrison RD, Yang P, Yang D-R. 2011. New insight into the phylogenetic and biogeographic history of genus Ficus: Vicariance played a relatively minor role compared with ecological opportunities and dispersal. J Syst Evol. DOI: 10.1111/j.1759-6831.2011.00155.x.

Zhang Q, Onstein RE, Little SA, Sauquet H. 2019. Estimating divergence times and ancestral breeding systems in Ficus and Moraceae. Ann Bot 123 (1): 191-204. DOI: 10.1093/aob/mcy159.

Zhang Z, Wang X-M, Liao S, Zhang J-H, Li H-Q. 2020. Phylogenetic reconstruction of Ficus subg, Synoecia and its allies (Moraceae), with implication on the origin of the climbing habit. Taxon DOI: $10.1002 / \operatorname{tax} 12282$ 